\title{
Kinematics of kiloparsec-scale jets
}

\author{
R. A. Laing ${ }^{1}$ and A. H. Bridle ${ }^{2}$ \\ ${ }^{1}$ ESO, Karl-Schwarzschild-Straße 2, D-85748 Garching-bei-München, Germany \\ email: rlaing@eso.org \\ ${ }^{2}$ NRAO, 520 Edgemont Road, Charlottesville, VA 22903-2475, USA \\ email: abridle@nrao.edu
}

\begin{abstract}
It has long been known that kiloparsec-scale jets in radio galaxies can be divided into two flavours: strong (found in powerful sources, narrow and terminating in compact hot-spots) and weak (found in low-luminosity sources, flaring, unable to form hot-spots and terminating in diffuse lobes or tails). Both flavours are initially relativistic, but weak jets decelerate to sub-relativistic, transonic speeds by entraining external gas while strong jets remain relativistic and supersonic until they terminate. Much is now known about the kinematics of weak-flavour jets, which can be modelled as intrinsically symmetrical, decelerating relativistic flows, and we summarize the results of our work in this area. Strong-flavour jets are relatively faint and narrow, so it has hitherto proved difficult to obtain the necessary deep, transverse-resolved images in total intensity and linear polarization. The spectacular jets in the radio galaxy NGC 6251 appear to represent a transition case between weak and strong flavours: the jets show no clear evidence for deceleration, but are relatively wide. VLA observations hint at transverse velocity structure with a very fast (Lorentz factor > 5) spine surrounded by a slower shear layer. New observations with the upgraded VLA should be able to test this picture.
\end{abstract}

Keywords. galaxies: jets, radio continuum: galaxies, magnetic fields

\section{Objectives}

Estimation of the flow parameters of extragalactic jets is notoriously difficult, mostly because neither of the dominant radiation processes - synchrotron and inverse Compton - provide many diagnostics. This paper summarizes recent progress in measuring the flow parameters for kiloparsec-scale jets in low-luminosity radio galaxies and previews an attempt to apply the same methods to more powerful jets using the upgraded VLA.

The principal questions we would like to answer are as follows.

(a) What are the jet velocity fields? Why do some jets expand rapidly ("flare") and decelerate, while other remain collimated and fast?

(b) What is the topology of the magnetic field? Is it globally ordered or disordered on small scales, but anisotropic? In either case, how do the ratios between longitudinal, radial and toroidal components vary along and across the jets? What is the field strength and how does it vary along the jet?

(c) What are jets made of? How do the proportions of electrons, positrons, protons and entrained thermal plasma vary with distance from the active galactic nucleus (AGN)?

$(d)$ What are the high-energy radiation mechanisms in jets? How are radiating particles accelerated?

(e) How are jets confined at various distances: by gas pressure, magnetic fields or not at all?

$(f)$ What are the mass, energy and momentum fluxes of jets? To what extent is jet energy and momentum transferred to the external environment? How is the environment affected? 
This paper is concerned primarily with questions (a) and (b), but makes brief reference to some of the other topics.

It has long been known that radio emission from AGN jets can be divided into strong and weak "flavours" (Bridle 1984). Strong-flavour jets are usually one-sided ( $>4: 1$ ) and well-collimated over their whole lengths. They often terminate in the compact hot spots characteristic of FR II morphology (Fanaroff \& Riley 1974). There is good qualitative evidence for relativistic flow in strong-flavour jets on kiloparsec scales (e.g. from depolarization asymmetry; Laing 1988) but the speeds are controversial. If X-ray emission from large-scale jets in quasars is produced by beamed inverse Compton scattering of cosmic microwave background photons, then the jets must have very high bulk Lorentz factors $\Gamma \approx 10$ (Tavecchio et al. 2000; Celotti, Ghisellini \& Chiaberge 2001). On the other hand, the distribution of jet/counter-jet sidedness ratios in FR II radio galaxies and quasars implies that $\Gamma \approx 1.2-1.4$ (Wardle \& Aaron 1997; Mullin \& Hardcastle 2009). The most obvious way to reconcile these two values is if the jets have fast $(\Gamma \approx 10)$ spines surrounded by much slower shear layers, but there is as yet little direct evidence for this hypothesis.

The speeds of weak-flavour jets are much better determined. These jets are found exclusively in sources with FR I morphology. They are initially asymmetric, but tend to symmetrize far from the nucleus after flaring and fading. The qualitative picture is that they are initially relativistic, but decelerate to subrelativistic speeds on kiloparsec scales, and we have demonstrated that detailed velocity fields can be estimated by fitting models of intrinsically symmetrical relativistic flows to deep, high-resolution radio observations.

In this paper, we describe our modelling method (Section 2) and its application to weak-flavour jets (Section 3). In Section 4, we show initial results for a transition case between weak and strong flavours and outline the prospects for future work with the Karl G. Jansky Very Large Array.

\section{Jet models}

This section summarizes the modelling method originally developed by Laing \& Bridle (2002a). A comprehensive overview of the technique and results is given by Laing \& Bridle (2014). We define the jet flow velocity to be $\beta c$, so that the bulk Lorentz factor is $\Gamma=\left(1-\beta^{2}\right)^{-1 / 2}$. We assume that the emission mechanism is optically-thin synchrotron radiation from electrons having an energy distribution $n(E) d E=n_{0} E^{-q} d E$ with $q=$ $2 \alpha+1 . \alpha$ is the spectral index $\left[I(\nu) \propto \nu^{-\alpha}\right]$.

We first make deep, high-resolution radio images in Stokes $I, Q$ and $U$ at frequencies sufficiently high that the effects of Faraday rotation can be accurately corrected: we have typically used multi-configuration VLA images at 5.0 and $8.4 \mathrm{GHz}$, with resolutions from $0.25-2.35$ arcsec and noise levels between 4 and $10 \mu \mathrm{Jy} /$ beam. Our fundamental assumption is that jets can be adequately approximated as intrinsically symmetrical, axisymmetric, relativistic, smooth flows. In that case, any observed differences between the approaching and receding jets must be a result of aberration. For a pair of cylindrical jets with uniform velocity $\beta$ inclined by an angle $\theta$ to the line of sight and emitting isotropically in the rest frame, the observed ratio of total intensity from the approaching (main) and receding (counter) jets is

$$
\frac{I_{\mathrm{j}}}{I_{\mathrm{cj}}}=\left(\frac{1+\beta \cos \theta}{1-\beta \cos \theta}\right)^{2+\alpha} .
$$

We cannot determine $\beta$ and $\theta$ independently from this ratio alone. In general, however, 
emission is anisotropic in the rest frame and the angular dependences are different for the three Stokes parameters. We effectively observe the main and counter-jets at different angles to the line of sight $\theta_{\mathrm{j}}^{\prime}$ and $\theta_{\mathrm{cj}}^{\prime}$, respectively, where in the uniform-velocity case:

$$
\begin{aligned}
\sin \theta_{\mathrm{j}}^{\prime} & =[\Gamma(1-\beta \cos \theta)]^{-1} \sin \theta, \\
\sin \theta_{\mathrm{cj}}^{\prime} & =[\Gamma(1+\beta \cos \theta)]^{-1} \sin \theta .
\end{aligned}
$$

Fitting simultaneously to total intensity and linear polarization then provides enough information to determine $\beta$ and $\theta$ provided that we can also fit for the transverse variation of the magnetic-field structure in the rest frame. To do this, we need good transverse resolution and high signal-to-noise.

Our approach is to construct parameterized models of four sets of quantities that between them determine the jet emission, as follows.

(a) Geometry: the shapes of the outer boundary and flow streamlines and the inclination, $\theta$.

(b) Velocity: longitudinal and transverse gradients.

(c) Emissivity function: the variation of $n_{0} B^{1+\alpha}$ along and across the jets, where $B$ is the rms magnetic field strength.

(d) Field ordering: the variation of the fractional field components in the longitudinal, toroidal and radial directions, $\left\langle B_{\mathrm{l}}^{2} / B^{2}\right\rangle^{1 / 2},\left\langle B_{\mathrm{t}}^{2} / B^{2}\right\rangle^{1 / 2}$ and $\left\langle B_{\mathrm{r}}^{2} / B^{2}\right\rangle^{1 / 2}$, respectively.

We optimize the model parameters using a downhill simplex algorithm to minimize $\chi^{2}$ between model IQU (convolved to the appropriate resolution) and the observed images.

\section{Models of weak-flavour jets}

\subsection{Model fits}

We have so far observed and modelled ten low-luminosity radio galaxies with weakflavour jets (Laing \& Bridle 2014). A comparison between our best-fitting model and the observations for the radio galaxy NGC 315 is shown in Fig. 1.

\subsection{Results}

The principal results from our observations and modelling are shown schematically in Figs 2(a) - (c).

We note first that the inclinations given by our modelling are consistent with those derived from independent indicators of orientation: fractional core flux density and Faraday rotation rms ratio (Laing \& Bridle 2014).

All of the jets we have modelled exhibit geometrically flaring regions in which the spreading rates first increase rapidly and then decrease. The jets eventually recollimate to form conical outer regions. They also show sudden brightness flaring following an initial dim, well-collimated region. Immediately downstream of the brightness flaring points and within the region of geometrical flaring, we often see bright, non-axisymmetric, knotty substructures (e.g. Fig. 1c): these define what we call the high-emissivity region.

The model velocity fields are shown in the right panels of Fig. 2. Where it first becomes measurable near the AGN, the outflow velocity has a mean value of $\beta=0.81$ with an rms dispersion of 0.08 . At this point, the transverse velocity profiles are consistent with constant values. Farther downstream, all ten jets we have modelled decelerate with increasing distance from the AGN. In at least six cases, there is good evidence that a transverse velocity gradient develops during deceleration: the outflow at the jet edges is slower than on-axis. 

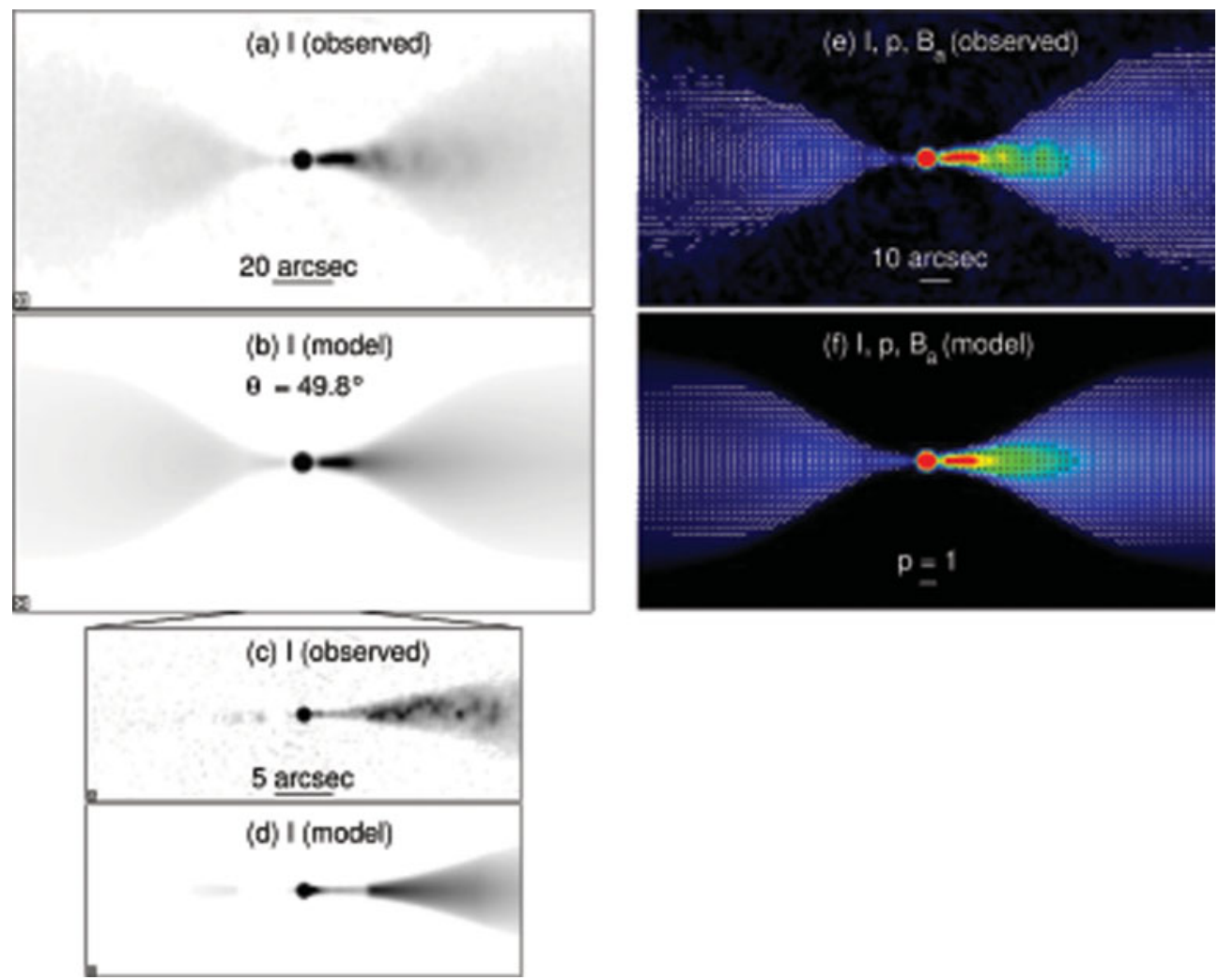

Figure 1. Comparison between model and observations for the jets in NGC 315. (a) observed and (b) model total intensity, $I$, at a resolution of 2.35 arcsec FWHM; (c) observed and (d) model $I$ at 0.6 arcsec resolution; (e) observed and (f) model images in which vectors with lengths proportional to the degree of polarization, $p=\left(Q^{2}+U^{2}\right)^{1 / 2} / I$ (Faraday depolarization is negligible), and directions along the apparent magnetic field are superposed on false-colour $I$. The apparent magnetic field is defined to be $\chi_{0}+\pi / 2$, where $\chi_{0}$ is the $\mathbf{E}$-vector position angle corrected for Faraday rotation. Further details are give in Laing \& Bridle (2014).

Jet magnetic fields are primarily longitudinal and toroidal, but not vector-ordered helices - helical fields would lead to unobserved asymmetries in the transverse profiles of both total intensity and linear polarization. The longitudinal component is strongest close to the AGN, but the toroidal component dominates at large distances (Fig. 3). The mean values of the rms on-axis fractional components (longitudinal:toroidal:radial) evolve from 0.78:0.55:0.29 close to the AGN to 0.55:0.80:0.23 after recollimation. At the edge of the jet, the corresponding evolution is from 0.62:0.61:0.50 (nearly isotropic) to 0.05:0.97:0.23 (almost purely toroidal). Although the evolution from longitudinal to transverse field is expected in an expanding flow, the quasi-one-dimensional flux-freezing approximation predicts a much more rapid transition from longitudinal to transverse field than we infer for any of the sources we have modelled.

The geometrically flaring regions, once scaled by the recollimation distance $r_{0}$ (Fig. 2a) have remarkably similar shapes and are homologous structures, in the sense that the characteristic scales for the evolution of velocity, emissivity function and magnetic field scale linearly with $r_{0}$. The brightness flaring point marks a discontinuity in some combination of speed and rest-frame emissivity function, located at $\approx 0.1 r_{0}$. The high-emissivity region runs from $\approx 0.1 r_{0}$ to $\approx 0.3 r_{0}$. Rapid deceleration starts midway along the high-emissivity 


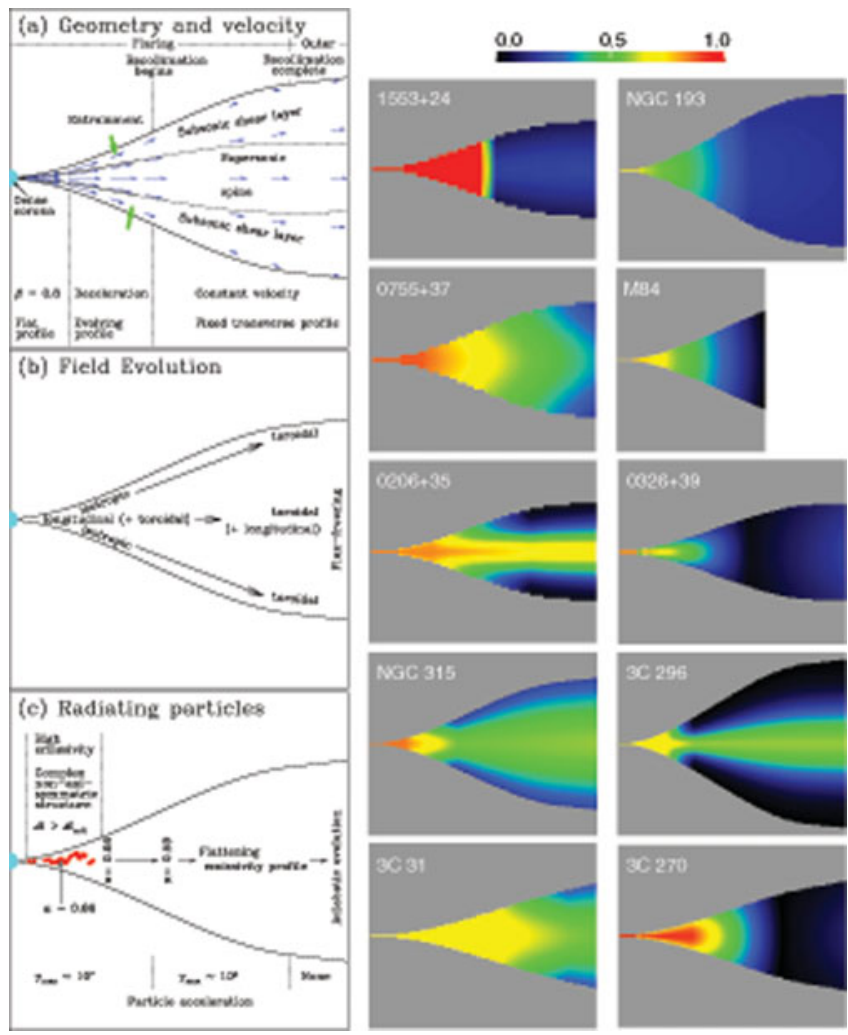

Figure 2. Left: Sketches showing the principal deductions from our model fits. (a) Geometry, velocity field and inferred boundary-layer entrainment. The blue arrows represent the velocity field. (b) Field-ordering parameters. (c) Emissivity function. The red pattern schematically represents the region in which bright knots are typically observed. $\gamma$ is the Lorentz factor of a radiating electron. Right: velocity, $\beta$, for a sample of ten FR I jets. Further details are given by Laing \& Bridle (2014).

region (at $\approx 0.2 r_{0}$ ) and lasts until $\approx 0.6 r_{0}$. Magnetic evolution begins near the AGN and essentially stops just after the end of the flaring region, at $\approx 1.1 r_{0}$ (where flux-freezing becomes a reasonable approximation). The end of rapid deceleration coincides accurately with the start of recollimation (i.e. where the spreading rate begins to decrease with distance.

In the flaring region, acceleration of ultrarelativistic particles is required to counterbalance the effects of adiabatic losses and account for observed X-ray synchrotron emission, but the brightness evolution of the outer jets is consistent with adiabatic losses alone. We find that the radio spectra of the jets always flatten slightly with increasing distance from the nucleus between the brightness flaring point and the end of rapid deceleration, from $\langle\alpha\rangle=0.66 \pm 0.01$ over the high-emissivity region to $\langle\alpha\rangle=0.59 \pm 0.01$ both immediately after deceleration and in the outer (recollimation) region (Laing \& Bridle 2013). The corresponding energy indices are $q=2 \alpha+1=2.32$ and 2.18 , respectively. The steeper spectra close to the jet flaring points are associated with typical bulk flow speeds $\beta>0.5$. Particle acceleration with $q=2.32$ over the energy range corresponding to $\mathrm{GHz}$ radio emission (electron Lorentz factors $\gamma \sim 2000-30000$, assuming equipartition magnetic fields) is required in the high-emissivity region. The process must be capable of accelerating electrons to $\gamma \sim 10^{7}-10^{8}$ for bulk flow speeds $\beta>0.5$. During deceleration, there 


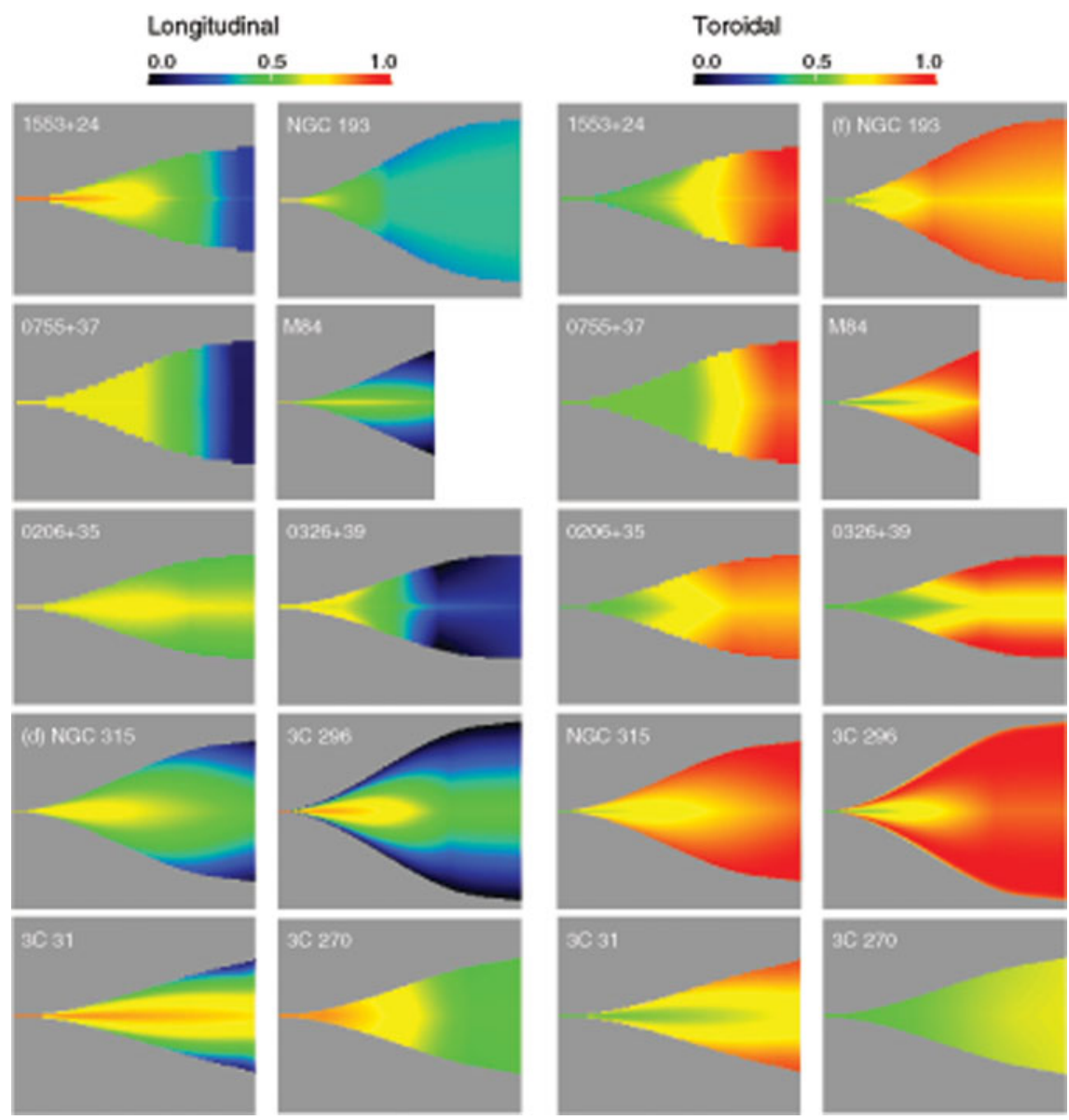

Figure 3. Fractional longitudinal and toroidal magnetic-field components from the models of Laing \& Bridle (2014). Left: longitudinal component $\left\langle B_{1}^{2} / B^{2}\right\rangle^{1 / 2}$. Right: toroidal component $\left\langle B_{\mathrm{t}}^{2} / B^{2}\right\rangle^{1 / 2}$.

is a gradual transition to a characteristic energy index $q=2.18$. High-energy radiation is still produced, but is less prominent relative to the radio emission. Finally, there is little evidence for ongoing particle acceleration after recollimation.

Starting from our kinematic model, we have also addressed the dynamics of jet deceleration in 3C 31 using a conservation-law approach and the assumption that jets reach pressure equilibrium with the external medium at large distances from the AGN (Laing \& Bridle 2002b). This allowed us to derive the variation of internal density, mass flux, entrainment rate and Mach number along the jets. The inferred energy flux for each of the jets in $3 \mathrm{C} 31$ is $\approx 10^{37} \mathrm{~W}$.

\section{NGC 6251}

\subsection{VLA observations and models}

In order to make a start on the much more difficult problem of determining the velocity field in strong-flavour jets, we need to find sources where the radio emission from both jets can be resolved transversely with good signal-to-noise in linear polarization as well as total intensity. The most promising case is the giant radio galaxy NGC 6251, whose 

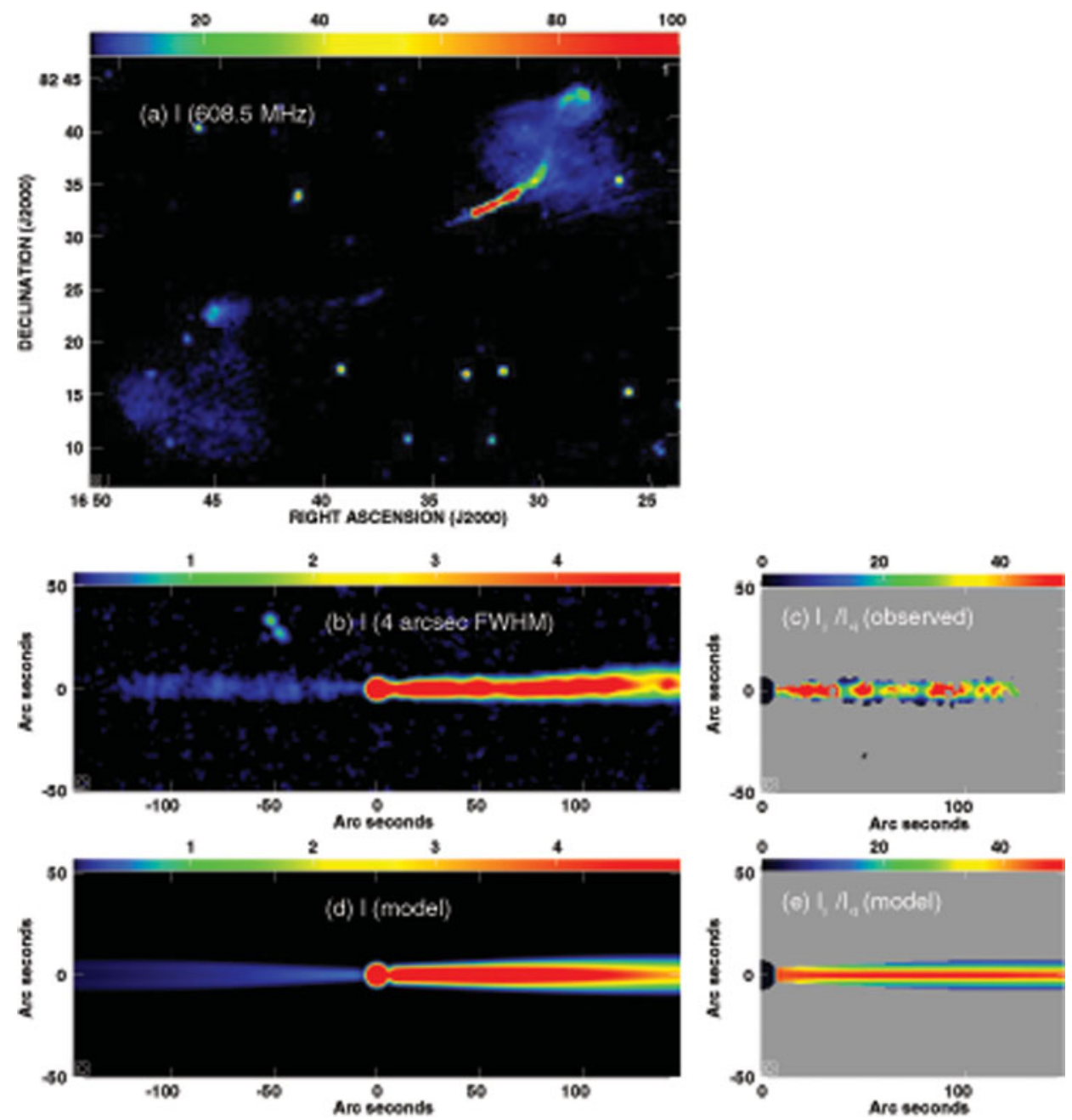

Figure 4. (a) - (c): observations of the radio galaxy NGC 6251. (a) WSRT observations of the entire source at a frequency of $609 \mathrm{MHz}$ (Mack et al. 1997). (b) Total intensity for the inner jets at $1665 \mathrm{MHz}$ derived from archival VLA observations. The resolution is $4 \operatorname{arcsec}$ FWHM. (c) Sidedness ratio $I_{\mathrm{j}} / I_{\mathrm{cj}}$, derived from (b). This is blanked (grey) where $I<3 \sigma$ in either jet. Note that the jet has been artificially "straightened" to produce this image. (d) and (e): total intensity and sidedness ratio for the relativistic jet model described in the text. This does not represent a fit to the data, but indicates that the observed asymmetries in the brightness distribution are roughly consistent with the model velocity field.

spectacular main jet was discovered by Waggett, Warner \& Baldwin (1977). The source (Fig. 4a) is one of the largest known (1.1 or $2.0 \mathrm{Mpc}$ in projection at $\mathrm{z}=0.0247)$.

The main jet was studied in detail by Perley, Bridle \& Willis (1984), who also discovered a faint counter-jet, for which a deep image at 10 -arcsec resolution was presented by Jones et al. (1986). We have reprocessed two long VLA archive datasets to produce the 4-arcsec FWHM image at $1665 \mathrm{MHz}$ shown in Fig. 4(b), giving the first width-resolved image of the counter-jet. Fig. 4(c) shows the distribution of the jet/counter-jet sidedness ratio $R=I_{\mathrm{j}} / I_{\text {cj }}$, derived by dividing the new image by a copy of itself rotated through $180^{\circ}$ (we have artificially straightened out $2^{\circ}$ bends in both jets before dividing these images). The sidedness ratio image clearly shows transverse structure in $R$ wherever the jets are 
adequately resolved (at distances $>40 \operatorname{arcsec}$ from the core). $\langle R\rangle \approx 40$ on-axis and $\approx 15$ at the edges. This is prima facie evidence for a transverse velocity gradient, just as in the weak-flavour jets, but with more extreme parameters. We see little evidence for large-scale sidedness variations along the jet, although analysis is complicated by fluctuations due to knots on both sides. The contrast between centre and edge is probably underestimated as the jets are only marginally resolved.

In Figs 4(d) and (e), we show the results from our code for a simple high-velocity model. This is not a fit to the data (we have no polarization information, so such a fit would not in any case be adequately constrained), but does show the type of intrinsically symmetrical model that could reproduce the observed morphology in $I$. The central ("spine") velocity is $\beta=0.995(\Gamma=10)$ and the jets are inclined by 32.5 to the line of sight. The velocity profile is a truncated Gaussian with an edge velocity of $\beta=0.4$.

\subsection{Future prospects}

We have recently obtained new, deep VLA observations of NGC 6251 in the $5-7 \mathrm{GHz}$ frequency range. The beamwidth $(\approx 1 \operatorname{arcsec} \mathrm{FWHM})$ is small enough to resolve both jets transversely. We expect to be able to detect linear polarization in both jets at this frequency (where the effects of Faraday rotation are relatively small) allowing us to constrain the jet flow speed and the inclination and to test the hypothesis of a very fast on-axis flow. If the model shown in Figs 4(d) and (e) is appropriate, we would expect to see large differences between the transverse brightness profiles of the main and counter-jets (centre- and limb-brightened, respectively). If the field structure is qualitatively similar to that in weak-flavour jets (Fig. 3), then we would also expect the transition between longitudinal and transverse apparent magnetic field on-axis to occur much farther from the nucleus in the main jet than in the counter-jet.

As mentioned earlier, NGC 6251 is in some respects a transition case between weak and strong-flavour jets. The next step is to apply our technique to the small number of bright strong-flavour jets, of which Cygnus $\mathrm{A}$ is probably the best case.

\section{Acknowledgements}

The National Radio Astronomy Observatory is a facility of the National Science Foundation, operated under co-operative agreement by Associated Universities, Inc.

\section{References}

Bridle, A. H. 1984, AJ, 89, 979

Celotti, A., Ghisellini, G., \& Chiaberge. M. 2001, MNRAS, 321, L1

Fanaroff, B. L. \& Riley J. M. 1974, MNRAS, 167, 31P

Jones, D. L., et al. 1986, ApJ, 305, 684

Laing, R. A. 1988, Nature, 331, 149

Laing, R. A. \& Bridle, A. H. 2002a, MNRAS, 336, 328

Laing, R. A. \& Bridle, A. H. 2002b, MNRAS, 336, 1161

Laing, R. A. \& Bridle, A. H. 2013, MNRAS, 432, 1114

Laing, R. A. \& Bridle, A. H. 2014, MNRAS, 437, 2405

Mack, K.-H., Klein, U., O'Dea, C. P., \& Willis, A. G. 1997, A\& AS, 123, 423

Mullin, L. M. \& Hardcastle, M. J. 2009, MNRAS, 398, 1989

Perley, R. A., Bridle, A. H., \& Willis, A. G. 1984, ApJS, 54, 291

Tavecchio, F., Maraschi, L., Sambruna, R. M., \& Urry, C. M. 2000, ApJ, 544, L23

Waggett, P. C., Warner, P. J., \& Baldwin, J. E. 1977, MNRAS, 181, 465

Wardle, J. F. C. \& Aaron, S. E. 1997, MNRAS, 286, 425 